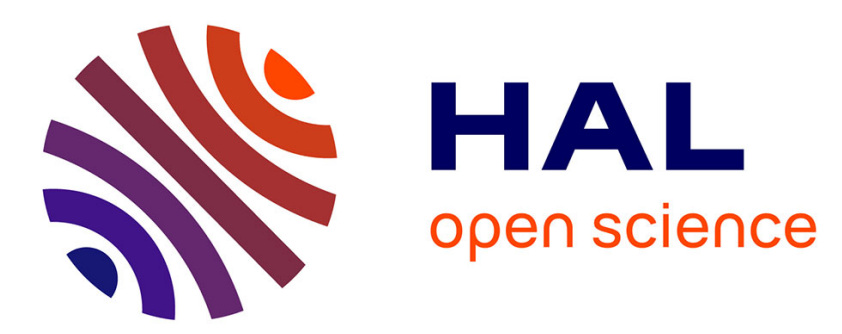

\title{
Formulation du champ dans un chenal acoustique sous-marin à l'aide des développements de Ludwig
}

R. Burvingt

\section{To cite this version:}

R. Burvingt. Formulation du champ dans un chenal acoustique sous-marin à l'aide des développements de Ludwig. Journal de Physique IV Proceedings, 1994, 04 (C5), pp.C5-1133-C5-1136. 10.1051/jp4:19945250 . jpa-00252939

HAL Id: jpa-00252939

https://hal.science/jpa-00252939

Submitted on 1 Jan 1994

HAL is a multi-disciplinary open access archive for the deposit and dissemination of scientific research documents, whether they are published or not. The documents may come from teaching and research institutions in France or abroad, or from public or private research centers.
L'archive ouverte pluridisciplinaire HAL, est destinée au dépôt et à la diffusion de documents scientifiques de niveau recherche, publiés ou non, émanant des établissements d'enseignement et de recherche français ou étrangers, des laboratoires publics ou privés. 


\title{
Formulation du champ dans un chenal acoustique sous-marin à l'aide des développements de Ludwig
}

\author{
R. BURVINGT
}

Le Centre Thomson d'Applications Radars, 6 rue Nieuport, BP. 86, 78143 Velizy-Villacoublay cedex, France

Résumé : Les développements asymptotiques uniformes de Ludwig sont considérés pour la description du champ dans un chenal acoustique sous-marin. Pour définir l'intégrale exprimant ce champ, on formule une expression analytique approchée de la solution de l'acoustique géométrique. Des rayons complexes peuvent être pris en compte dans la zone d'ombre des caustiques. On présente ensuite une méthode générale de calcul de cette intégrale.

Abstract : The Ludwig's uniform asymptotic developments are considered for description of underwater acoustical channels. The integral which gives the field is formulated with help of approached analytical expressions of the acoustical geometrical solution. Complex rays are taken into account in the shadow regions. A method of computation for this integral is presented here.

\section{INTRODUCTION.}

Dans [1] a été exposé le principe d'une méthode de description d'un chenal acoustique. Dans cette méthode sont pris en compte les développements asymptotiques uniformes de Ludwig [2]. Les calculs qui ont été mentionnés étaient relatifs à un chenal dont le profil d'indice est quartique (le carré de l'indice s'exprime à l'aide d'un polynôme de degré quatre fonction de l'immersion (cf. [3])).

Dans l'étude présentée ici, on montre comment cette méthode peut être employée dans une description d'un chenal dont le profil d'indice est quelconque. On considère une source ponctuelle pouvant être directive et des chenaux stratifiés horizontalement. Le point de départ de la méthode est une représentation du champ sous la forme intégrale suivante :

$$
u=\int_{C} g(\vec{x}, \xi) e^{i k \varphi(\vec{x}, \xi)} d \xi,
$$

où, rappelons le, la fonction $\varphi$ est un polynôme de degré $n+1$ en $\xi$, si $n$ rayons de l'acoustique géométrique concourrent au point d'observation $\vec{x}$. g est un polynôme de degré $n-1$ en $\xi$. La suite des $n$ valeurs de $\varphi$ aux points selle de l'intégrale est identique, à des permutations près, à la suite des valeurs des phases de l'acoustique géométrique relatives aux n rayons concourrants.

Le développement des calculs nécessite dans un premier temps, une détermination des solutions de l'acoustique géométrique. Comme nous l'avons indiqué dans [1,3], pour obtenir ces solutions dans la zone d'ombre des caustiques, des rayons complexes peuvent être considérés (rayons lancés sur la source 
pontuelle avec un angle complexe). Pour définir un rayon complexe en un point d'observation situé dans une zone d'ombre, un prolongement dans le domaine complexe de l'équation des rayons réels doit être effectué. Cependant un tel prolongement n'est possible que si les rayons réels sont exprimés sous une forme analytique. Dans [1] une telle forme pouvait être prise en compte étant donné qu'un profil quartique était retenu. Les rayons s'exprimaient à l'aide de fonctions et d'intégrales elliptiques. Dans le cas présent d'un chenal de profil d'indice quelconque, on montre dans ce qui suit qu'une expression analytique approchée des rayons peut être obtenue si des développements en série de Fourier sont considérés.

La solution de l'acoustique géométrique relative aux rayons réels et complexes pouvant être déterminée, le calcul des coefficients de $\varphi$ et $g$ et puis l'évaluation de (1) doivent être entrepris. Le calcul de ces coefficients peut être exact si $n \leq 3$ [3]. Lorsque $n>3$, on montre ici que l'on peut se ramener pour l'évaluation de (1) qu'à des considérations de cas où $\mathrm{n} \leq 3$. Ceci est possible si l'intégrale précédente est représentée sous la forme d'une somme d'intégrales plus élémentaires.

\section{EXPRESSION DES RAYONS EN TERME DE SERIE DE FOURIER.}

Un rayon émis sous un angle $\theta_{\mathrm{i}}$ donné, à une position $\mathrm{z}_{\mathrm{o}}$ de la source également donnée, possède une excursion verticale que l'on peut exprimer en fonction de la distance horizontale $r$ sous la forme

$$
z=A_{i, o}+\sum_{m=1}^{M} A_{i, m} \operatorname{cosin}\left(\omega_{i} r+g_{i}\right)
$$

La précision de cette approximation dépend de la valeur de l'entier $M$. Les coefficients $A_{i, m}$, $\omega_{i}$ et $g_{i}$ sont calculés de manière approchée après un découpage de l'intervalle de profondeur, dans lequel est situé le chenal, en sous-intervalles dans lesquels les variations du carré de l'indice $n^{2}(z)$ sont approximativement linéaire. On note que pour déterminer ces coefficients des sous-intervalles, dans lesquels les variations de $n^{2}(z)$ sont approximativement paraboliques, peuvent également être considérés. Le découpage peut alors être moins fin.

Par ces calculs on fait correspondre, à une suite de valeurs discrètes $\theta_{i}$, les suites de valeurs $A_{i, m}$ $(\mathrm{m}=0, M), \omega_{\mathrm{i}}$ et $\mathrm{g}_{\mathrm{i}}$. A partir de ces dernières suites on peut construire des fonctions continues $\mathrm{A}_{\mathrm{m}}\left(\mathrm{z}_{\mathrm{o}}, \theta\right)$, $\omega(\theta)$ et $\mathrm{g}\left(\mathrm{z}_{\mathrm{o}}, \theta\right)$ en utilisant, par exemple, la méthode des moindres carrés. Ces fonctions, qui sont alors des polynômes en $\theta$, peuvent approximativement prendre lorsque $\theta=\theta_{\mathrm{i}}$, les valeurs $\mathrm{A}_{\mathrm{i}, \mathrm{m}}$, $\omega_{\mathrm{i}}$ et $\mathrm{g}_{\mathrm{i}}$ et, des valeurs d'interpolation lorsque $\theta \neq \theta_{\mathrm{i}}$.

On peut donc calculer les rayons de manière approchée à l'aide d'une expression analytique en $\theta$ de la forme :

$$
z=A\left(z_{o}, \theta\right)+\sum_{m=1}^{M} A_{m}\left(z_{o}, \theta\right) \cos m\left(\omega(\theta) r+g_{o}\left(z_{o}, \theta\right)\right)
$$

Si l'on retient, par exemple, la bathycélérimétrie représentée sur la figure 1 et une position de source également représentée sur celle-ci, à partir de cette formule (3) on peut obtenir les rayons représentés sur la figure 2. On a constaté que ces derniers peuvent être très proches des rayons calculés à l'aide de méthodes plus classiques. On retient 5 termes dans la série de Fourier. 


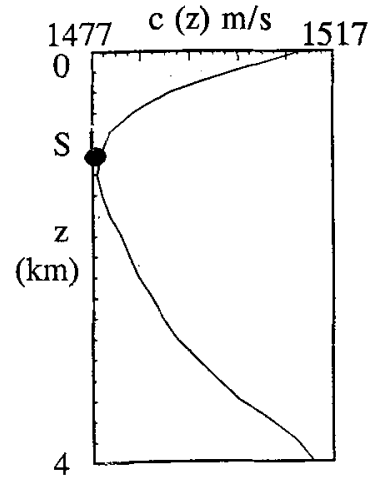

Fig. 1 : Bathycélérimétrie

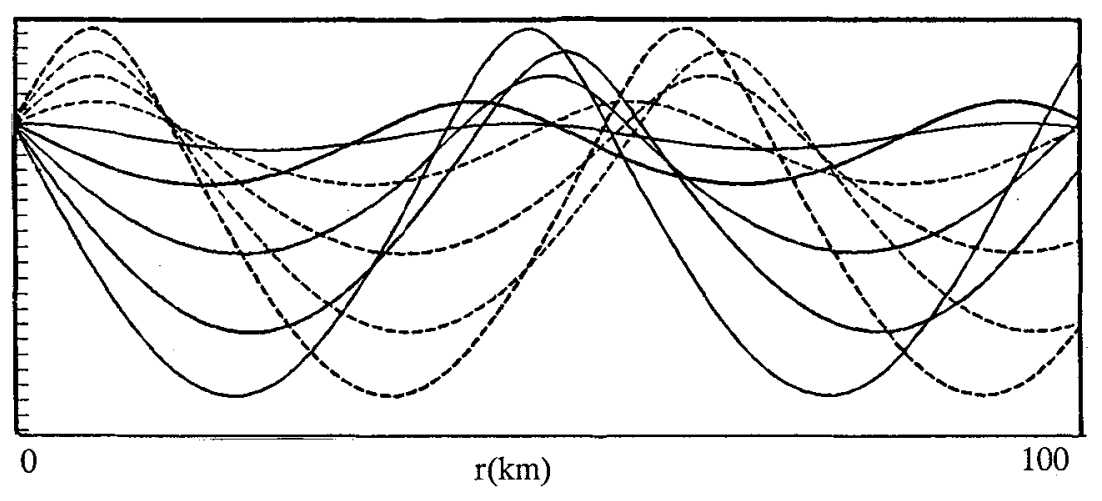

Fig. 2 : Diagramme de rayons $\left(\theta \in\left[-5,5^{\circ} ; 5,5^{\circ}\right]\right)$

Cette expression (3) semble pouvoir être employée pour bon nombre de chenaux lorsque la source possède une petite ouverture (de l'ordre de $10^{\circ}$ à $20^{\circ}$ ). Les polynômes, auxquels conduit la méthode des moindres carrés, ne sont pas alors de degré trop élevé.

Elle peut être retenue pour déterminer les caustiques et ensuite les angles complexes sous lesquels sont émis les rayons complexes passant aux points $(r, z)$ ( $r$ et $z \in \mathbb{R}$ ) des zones d'ombre. De ces angles complexes on peut déduire des phases et des amplitudes complexes de l'acoustique géométrique dans ces zones d'ombre. Ces phases et ces amplitudes peuvent être utilisées pour définir les fonctions $\varphi$ et $g$ de (1) $[4]$.

\section{EXPRESSION DU CHAMP EN TERME DE FONCTIONS INCOMPLETES.}

Dans [1], une expression de l'intégrale (1) sous la forme d'une somme d'intégrales plus élémentaires avait été mentionnée dans le cas où cinq rayons concourrent au point d'observation. Une telle formulation peut être généralisée au cas d'un plus grand nombre de rayons.

L'axe réel est choisi comme contour d'intégration. A un rayon réel de numéro $\mathrm{m}$, on associe un point selle réel $\xi_{\mathbf{R}, \mathrm{m}}\left(\xi_{\mathbf{R}, \mathbf{m}} \in \mathbb{R}\right)$. A deux rayons complexes conjugués, on associe un couple de points selle complexes conjugués (situés symétriquement de part et d'autre de l'axe réel). A ce couple on fait correspondre un point d'inflexion $\xi_{\mathrm{I}, \mathrm{m}}\left(\xi_{\mathrm{I}, \mathrm{m}} \in \mathbb{R}\right)$ de la fonction $\varphi(\xi)$ lorsque $\xi \in \mathbb{R}$. En faisant intervenir une hypothèse d'une bijection entre $\xi$ et l'angle $\theta$ de lancement des rayons, on définit une ordonnance des $\xi_{\mathrm{R}, \mathrm{ml}}$ et $\xi_{1, \mathrm{~m}}$ sur l'axe réel. Cet axe peut être scindé en intervalles qui contiennent (i) soit un, deux ou trois $\xi_{R, m}$, (ii) soit un $\xi_{\mathrm{I}, \mathrm{m}}$, (iii) soit un $\xi_{\mathrm{I}, \mathrm{m}}$ et un $\xi_{\mathbf{R}, \mathrm{m}}$. Compte tenu de l'introduction de tels intervalles au nombre de 1 , le champ s'écrit sous la forme :

$$
u=\sum_{j=1}^{l} u_{j},
$$

où les termes $u_{j} s^{\prime}$ expriment à l'aide (i) soit de fonctions de Fresnel (un seul $\xi_{\mathbf{k}, \mathrm{m}}$ appartient à l'intervalle j), (ii) soit de fonctions d'Airy incomplètes (il existe soit un $\xi_{1, m}$ ou, soit deux $\xi_{\mathbf{R}, \mathrm{m}}$ dans l'intervalle j) et (iii) soit de fonctions de Pearcey incomplètes (ils existent soit un $\xi_{1, m}$ et un $\xi_{\mathbf{R}, \mathrm{m}}$, ou soit trois $\xi_{\mathbf{R}, \mathrm{m}}$ dans l'intervalle $\mathrm{j}$ ). 
Ces fonctions s'introduisent moyennant un changement de variable d'intégration. Dans un intervalle donné la phase est un polynôme (fonction de la nouvelle variable) dont les coefficients sont déterminés exactement. L'amplitude peut y être déterminée approximativement à partir des équations linéaires satisfaites par les coefficients du polynôme g de (1) [4] et de conditions de raccordement aux limites de cet intervalle.

\section{CONCLUSION.}

Par l'introduction d'expressions analytiques approchées, les rayons complexes, devant être pris en compte dans la zone d'ombre des caustiques, peuvent être déterminés. Ils permettent de formuler l'intégrale exprimant le champ pour laquelle une méthode de calcul a été définie. Le champ dans un chenal acoustique peut donc être alors évalué. Des applications pratiques seront présentées ultérieurement.

\section{REFERENCES.}

[1] R. BURVINGT, "Description d'un chenal acoustique sous-marin à partir de l'approche de Ludwig", Deuxième Congrés Français d'Acoustique, Volume II, J. Roux Ed., Arcachon - France - avril 1992 (Les Editions de Physique, Les Ulis, 1992) pp. 1049-1052.

[2] D. LUDWIG, Comm. Pure Appl. Math., 19 (1966) 215-250.

[3] R. BURVINGT, "Propagation dans un chenal sous-marin profond", Premier Congrés Français d'Acoustique, Volume II, P. Filippi, M. Zakharia ed., Lyon - France, février 1990, (Les éditions de Physique, les Ulis, 1990) pp. 1033-1036.

[4] L. SALTIEL, Revue du Cethedec, 48 (1976) 35-74. 